\title{
Calculations of Magnetic Field for the End Design BNL-49365 of the RHIC Arc Dipole*
}

\author{
S.A. Kahn, R.C. Gupta, A.K. Jain, G.H. Morgan, and P.A. Thompson \\ RHIC Project \\ Brookhaven National Laboratory, Upton, NY 11973
}

\begin{abstract}
This paper describes the results of a $3 D$ calculation of the magnetic field in the end region of the RHIC arc dipole magnet. Multipole harmonics are obtained in the end regions of the magnet. Comparisons of these harmonics are made to the measurements of harmonics on the actual magnets.
\end{abstract}

\section{INTRODUCTION}

This paper summarises the 3D calculations performed on the ends of the RHIC dipole magnet. The design of the ends depends both on magnetic and mechanical considerations. The magnetic objectives are to minimise integrated harmonics and to reduce fields at the conductors in the end region. The mechanical consideration is to minimise the strain energy of the cable in the end configuration. The cable can be viewed as a ribbon with an easy direction (perpendicular to the ribbon plane) and a hard direction (in the plane of the ribbon) of bend. Strain would be minimised if the cable would bend only in the easy direction. In the end, the cable traverses a path where the outer edge is on a cylinder. On the surface which describes the mid-plane of the ribbon the edges of the cable should ideally be geodesic of the surface. The mathematical treatment of the end design is patterned after Bossert et $\mathrm{a}^{\mathbf{1}}$. The outer and inner edges are assumed to be ellipses whenthe cylinder on which it lies is unrolled. This procedure of using developed contours for the cable has made the winding of the ends reasonably easy.

The RHIC dipole magnet has a single layer with four blocks in the 2D cross section. Spacers are placed in the ends to spread the positioning of the various turns so as to control the field harmonics. This is performed by a minimisation technique. Fig. 1 shows a projection of the outer edge of the return end of the coil onto a plane. The four blocks merge into seven regions in the end with different spacer sises.

Calculations of the magnetic fields can be performed hased on this model of the ends. Results using the BiotSavart integration on the conductors are given in section 2. Comparisons are made to measured data for these magnets. Section 3 describes similar calculations that are in progress made with the $3 D$ finite element program TOSCA $^{2}$ which takes into account the iron saturation. Also the TOSCA calcuia:ions will be used to study the peak field in the vicinst the conductor.

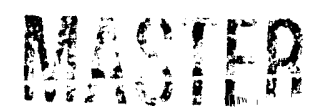

\section{CONDUCTOR INTEGRATION}

The field at a point in space is obtained by integrating the Biot-Savart relation over the entire conductor path. The coils consist of 32 turns of cable in each half. Each turn is represented by a straight section plus an ellipse in the unrolled cylinder plane for the end region. For field calculations each turn is subdivided by 3 in thickness and by 5 in width with each subdivision being approximated by a filament at its center. The infinite permeability iron is approximated by including the image current to each filament in the iron cylinder. The method of images is not strictly correct since the non-axial components of current do not form images in the cylindricai geometry. However, the non-axial components do not contri'sute to the field harmonics.

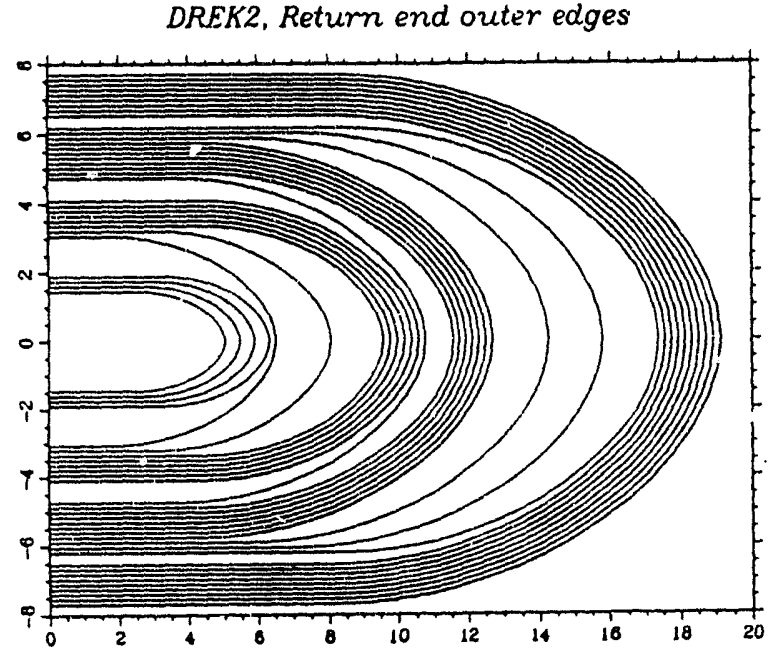

Figure 1: Outer edge of the RHIC dipole return end.

The field is Fourier analysed in terms of harmonics defined as follows:

$$
B_{y}+i B_{x}=10^{-4} \times B_{0} \sum_{n=0}^{\infty}\left[b_{n}^{\prime}+i a_{n}^{\prime}\right]\left[\frac{x+i y}{R}\right]^{n}
$$

where $B_{x}$ and $B_{y}$ are the field components at $(x, y), R$ is the reference radius chosen to be $25 \mathrm{~mm}$ and $B_{0}$ is the $2 D$ field at the center of the magnet. $b_{n}^{\prime}\left(a_{n}^{\prime}\right)$ represents the normal (skew) field harmonics. Fig. 2 shows the calculated normal field harmonics as a function of axial position in the end region of the return end (the end without the lead connections) of the magnet. The axial position is somewhat arbitrary with $=0$ approximstetnes. nISTFIEUTION! OF THIS DOCUMENT IS UN 


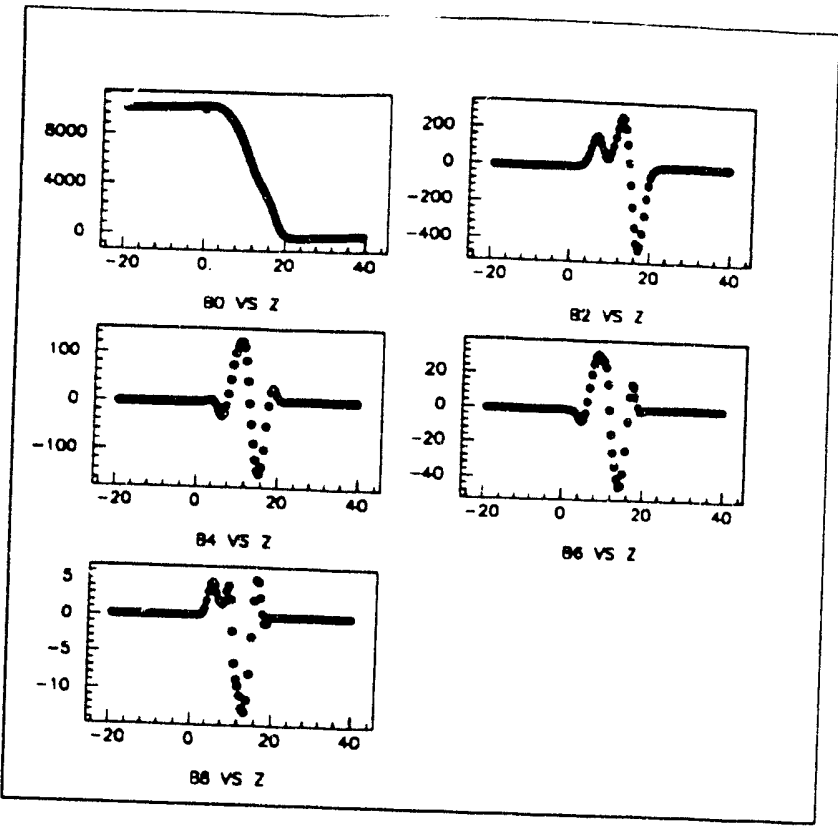

Figure 2: Calculated normal field harmonics for the RHIC dipole return end. Axial position is in $\mathrm{cm}$.

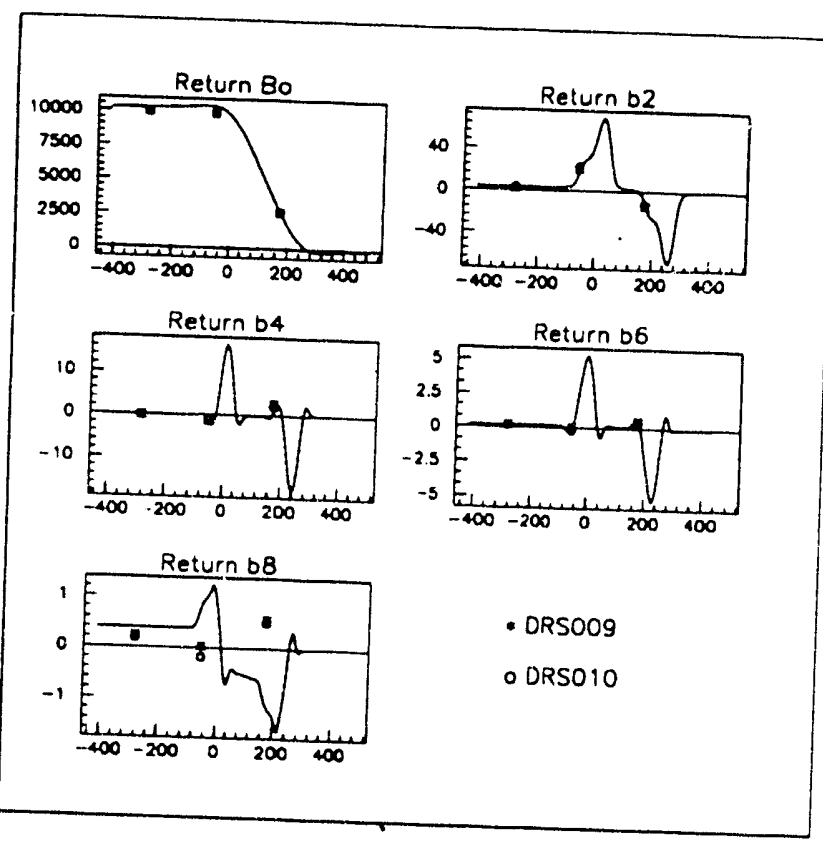

Figure 3: Return end normal harmonics convoluted with 9 inch rectangular function simulate the measurement if it were performed at that $z$ position. Corresponding harmonics for actual measurements are superimposed. The $z$ position is quoted in $\mathrm{mm}$ in this figure.

cm before the beginning of the curved part of the end. Negative numbers are inside the straight section of the magnet.

Measurements in the end region of a magnet are made with a $\theta$ inch long coil. The measuring coil is stepped in 9 inch $i$ ments through length of the magnet. A sequence of seven steps covers the length of the DRS009 and DRS010 short dipole magnets. To simulate the measurements, a convolution is made of the end calculation at a point $z$ with a 9 inch band centered around it. Fig. 3 and Fig. 4 show these convoluted calculations for the normal return end and lead end harmonics as the continuous curve. The non-convoluted end effect of the harmonics has an active range of about $15 \mathrm{~cm}$. When this active range is completely within the 9 inch $(22.9 \mathrm{~cm})$ band the convoluted harmonics are near iero since the ends were designed to have harmonies that integrate to sero. When upper and lower edges of the 9 inch band pass through the active range a positive and a negative reflection of the profile appear, separated by 9 inches. The measured 9 inch coil data from DRS009 and DRS010 at 2000 amps are also shown in Fig. 3 and Fig. 4. This current is chosen since it is below the turn-on of iron saturation effects and above most of the superconducting magnetization. The arbitrariness of the relative axial position between the measured and calculated harmonics is removed by matching $B_{0}$ at the most sensitive 9 inch measurement segment. Furthermore the calculated harmonics are corrected to match the measured harmonics at the position in the middle of the magnet. This is done since the crude representation of the iron in the calculation does not take into account the fine iron details such as notches and other cutouts present in the return yoke. The agreement between the calculation and measurements are remarkably good with the possible exception of $b_{8}$, however higher order harmonics are generally more difficult to calculate.

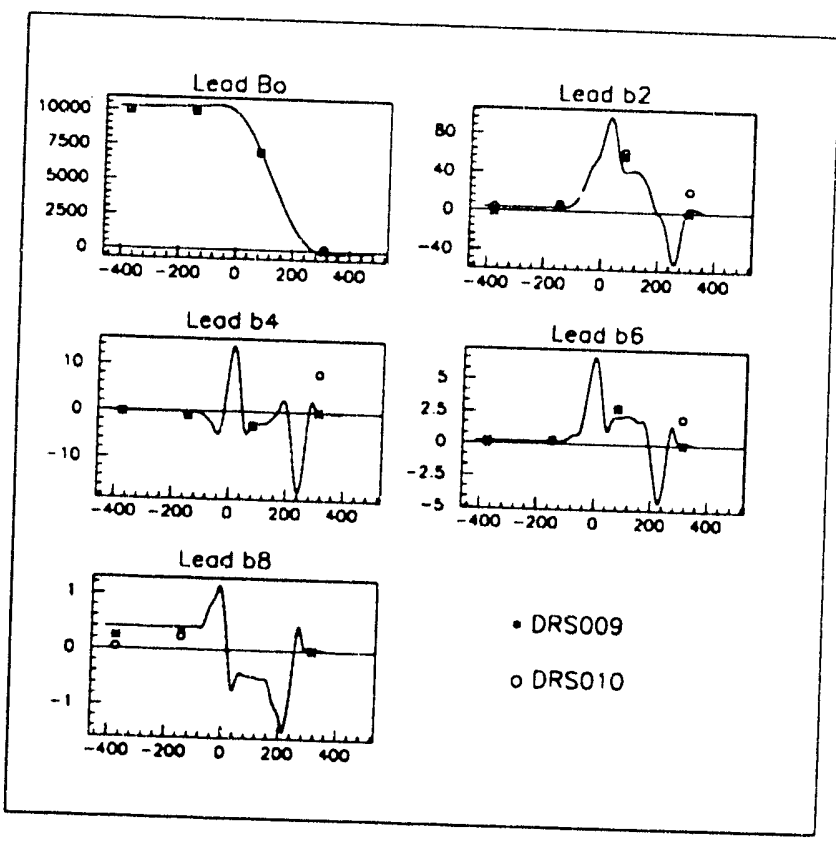

Figure 4: Lead end normal harmonics convoluted with 9 inch rectangular function simulate the measurement if it were performed at that $z$ position. Corresponding harmonics for actual measurements are superimposed. The $x$ position is quoted in $\mathrm{mm}$ in this figure. 
The end harmonics can be defined as

$$
b_{n}^{e n d}=b_{n}^{i n t}-b_{n}^{b o d y} \times L_{e f f}
$$

where the effective length, $L_{e f f}$, is $10^{-4} \times b_{0}^{\text {int }}$. This non-standard definition ${ }^{3}$ of end harmonics is used to allow the direct comparison of the calculation with the measured data. Using the $\boldsymbol{9}$ inch measurements the end harmonics can be separated into contributions from the lead and return ends. The lead and return end harmonics are considered spacially localized contributions to the accelerator lattice in addition to the $2 \mathrm{D}$ body harmonics, $b_{n}^{b o d y}$. Table 1 presents the return and lead end harmonics for DRS009 and DRS010 along with the calculated values. The agreement is reasonably good. The skew harmonies, $a_{n}^{e n d}$, are also given. The calculation of $a_{n}^{e n d}$ are made from the leads alone. The comparison indicates that these skew harmonics are associated largely to these leads. The leads in the measurement test apparatus are unfortunately positioned to give large harmonics. In the accelerator these leads are designed to have minimal effect.

Table 1: Integrated harmonics for return and lead ends. Results are listed as unit-meters.

\begin{tabular}{|c|c|c|c|c|}
\hline End & $\mathrm{n}$ & DRS9 & DRS10 & Calc \\
\hline Return & $b_{0}$ & 0.00 & 2.92 & 0.00 \\
& $b_{2}$ & 0.78 & 1.43 & 0.20 \\
& $b_{4}$ & 0.39 & 0.25 & -0.01 \\
& $b_{6}$ & 0.07 & 0.06 & 0.01 \\
& $b_{8}$ & -0.25 & -0.22 & -0.17 \\
& $a_{2}$ & 0.15 & 0.43 & \\
& $a_{4}$ & 0.08 & 0.22 & \\
& $a_{6}$ & 0.00 & 0.03 & \\
\hline Lead & $b_{0}$ & 0.00 & 2.79 & 0.00 \\
& $b_{2}$ & 13.53 & 14.37 & 10.12 \\
& $b_{4}$ & -0.83 & -0.79 & -0.55 \\
& $b_{6}$ & 0.63 & 0.65 & 0.51 \\
& $b_{8}$ & -0.23 & -0.23 & -0.15 \\
& $a_{2}$ & -7.53 & -7.93 & -7.41 \\
& $a_{4}$ & 1.81 & 1.65 & 1.26 \\
& $a_{6}$ & -0.75 & -0.71 & -0.28 \\
& $a_{8}$ & 0.22 & 0.21 & -0.05 \\
\hline
\end{tabular}

\section{CONCLUSIONS}

The good agreement of the low field measured harmonics with the calculations implies that the conductor geometry in the coil ends is as it should be. Currently in progress is an analysis of the RHIC dipole end using the saturable iron computer program TOSCA. This program solves Poisson's equations in $3 D$ using a finite element method. In this described by a constant perimeter conductor with a developed ellipse in the end region in a similar manner to that which was used in the previous section. The iron is a simple cylinder with the correct inner and outer yoke radii. Other structures in the iron are ignored since the segmentation available on a 3D mesh is not sufficient for such detail. The first part of the study is to understand the behavior of the field harmonics after the onset of iron saturation. The measured harmonics at high ficld show effects in the $b_{2}$ term due to saturation.

The second part of the study is to examine the field in the vicinity of the conductor in the end region. The peak field near a conductor can potentially cause a quench at that location. It is desirable that such high fields not be in the end region where the coil support may be less rigid. Differences of a few percent in field can be important.

\section{REFERENCES}

1. R.C. Bossert et al, Analytical Solutions to SSC Coil End Design, Supercollider I, (1990).

2. TOSCA is a computer code marketed by Vector Fields, Ltd.

3. Definition of the end harmonics used for field measurements are described by A.K. Jain and P. Wanderer in an internal note, MDN 482-11 (RHIC-MD-186).

"Work performed under Contract No. DE-AC02-76CH00016 with the U.S. Department of Energy.

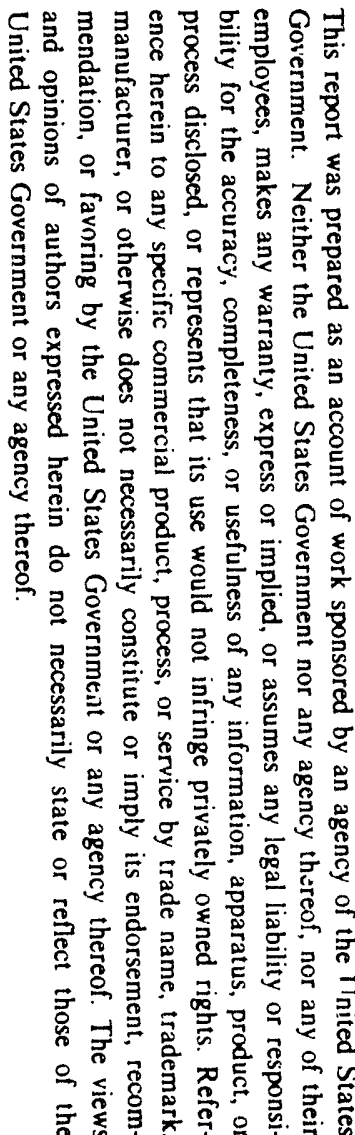



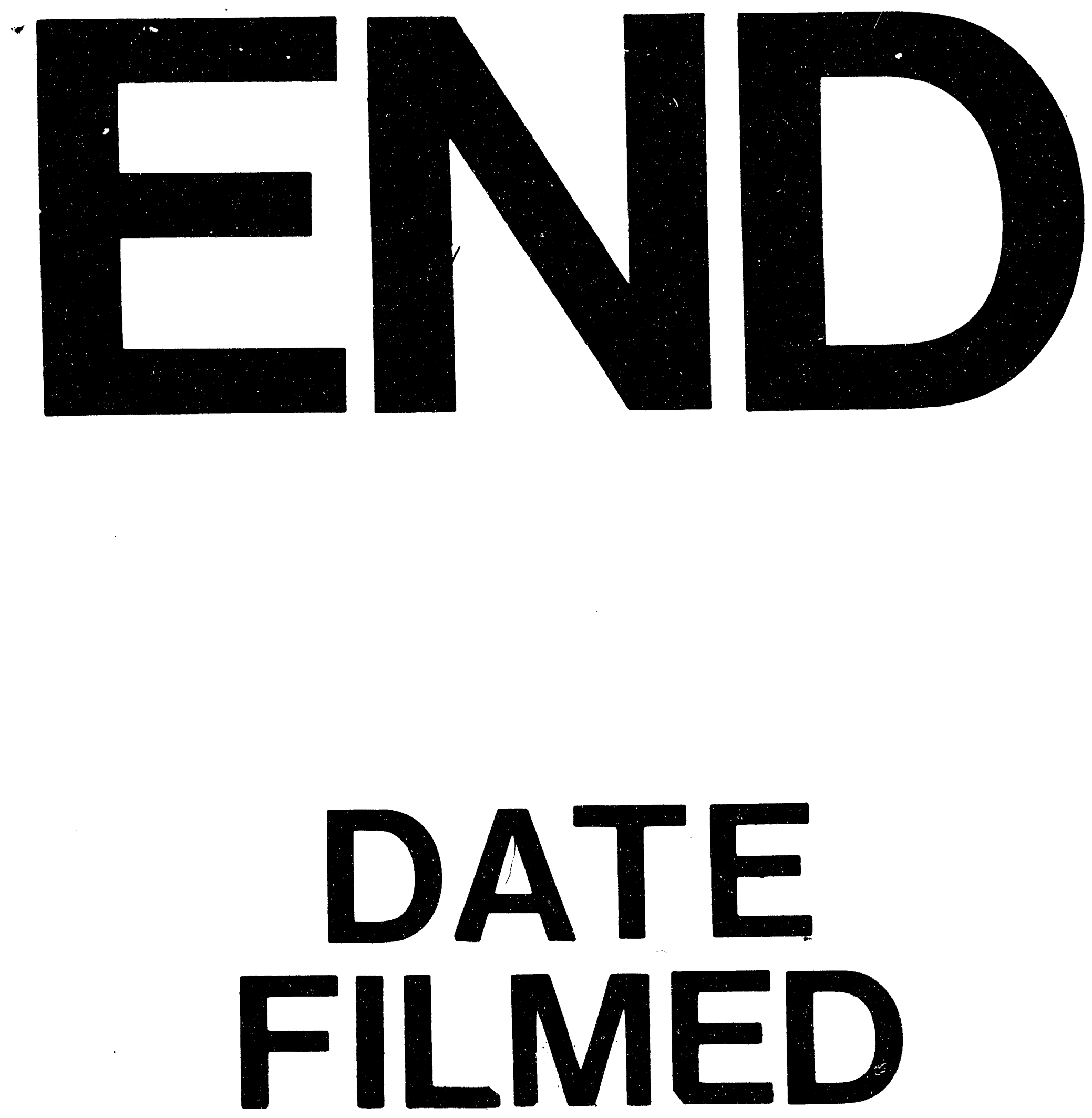

I

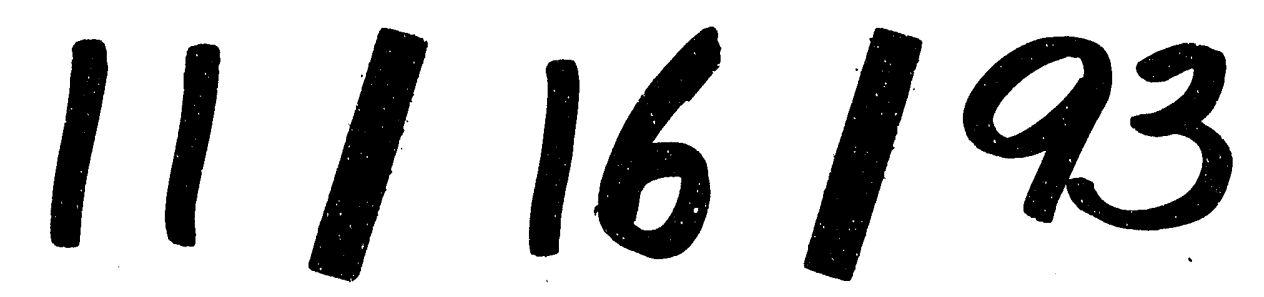


